\title{
Time Management Styles and Food Consumption: A Pilot Study of American and Chinese College Students
}

\author{
Tal Gordon and Napatsorn Firaporn
}

Tal Gordon (tgordon2@oswego.edu) is an undergraduate student majoring in marketing and finance and Napatsorn Jiraporn (napatsorn.jiraporn@oswego.edu) is an Assistant Professor at the School of Business, State University of New York at Oswego, 7060 Route 104, Oswego, NY 13126. Correspondence concerning this paper should be addressed to Napatsorn Jiraporn. We thank the anonymous reviewer and the editor for their constructive comments.

\begin{abstract}
This research investigates the relationship between time management styles and food consumption among college students in the U.S. and China. Prior research in consumer psychology suggests that time scarcity is a barrier to healthy food consumption. Thus, it was hypothesized that consumers who practice good time management skills should be able to make healthier food decisions. Moreover, we proposed and found that culture (American vs. Chinese) moderates the positive effect of time management on food choices. One hundred and twenty one American and
\end{abstract}


Chinese students participated in this study by completing an online survey and the findings show that American and Chinese participants differ in both their time management styles and eating motives. Specifically, Americans engaged more in short-range planning, reported greater time attitude, and greater preference for organized time than Chinese. Food choices among Americans are driven by liking, habits, convenience, and sociability factors whereas those among Chinese are driven by social norm and social image. Further analysis shows that as hypothesized, consumers with greater preference for organized time also selected healthier food choices. This positive effect of time management on food consumption is stronger among Chinese than Americans. The findings offer implications for health researchers, consumers and public policy makers.

Keywords: food consumption, food choices, time management, eating motives, cross-cultural consumer behaviors

DOI: http://dx.doi.org/10.15239/j.brcadvjb.2018.03.01.ja03

\section{INTRODUCTION}

Time is a salient component of life that everyone has the pleasure of encountering in some way, shape or form. Time is also a limited resource so time management became crucial to control to produce desired outcomes. Time management is often significant in reference to mismanaging it or misusing it (Britton and Tesser 1991). Topics related to time management are a prevalent area of research because it affects a multitude of factors in our everyday lives such as attitude, food consumption, academic performance, and stress level (Adam, and Jex 1999; Kaufman-Scarborough and Lindquist 1999; Misra and McKean 2000; Trueman and Hartley 1996). In the scholarly literature, researchers have paid attention to time management among college students but this is not a surprise given that 4 out of 5 college students in the U.S. work at least part-time while they are pursuing their higher education (Kingkade 2013). Navigating through studying, meeting paper deadlines 
and participating in extracurricular activities in addition to earning for their livings requires effective time management. Generally researchers agree that time management is positively related to academic achievement (Hoff Macan and Shahani 1990, Britton and Tesser 1991). However, the topic which has received little attention from researchers is how time management among college students affect other dimensions of their lives such as their food consumption and ability to maintain healthy lifestyle?

Only a handful of research has been conducted on the effects of time management and time perception on food consumption. Jabs and Devine (2006) presented an overview of time issues (e.g. time scarcity) related to food choices and consumption patterns. Specifically, the authors proposed that feeling of time scarcity may be associated with people's tendency to shorten their meal preparation time, speed up their food consumption, and promote ordering takeout or buying fast food. The authors argued that these food choices could be associated with unhealthy diets and possibly promote chronic health problems (e.g. diabetes, heart disease). Thus, the authors highlighted the need to empirically test the relationship between feeling of time scarcity and good choices. Later, Jabs et al. (2007) examined how employed mothers managed their time to provide meals for themselves and families. Through in-depth interviews, low-wage employed mothers expressed feeling of time scarcity and as a result, showed tendency to quickly prepare meals in order to continue to complete other tasks. Further empirical evidence was offered by Welch et al. (2008) which revealed that out of 1,580 women who participated in the study, $73 \%$ reported that time pressure is a barrier to healthy eating. They were less likely to meet the recommended amount of fruit and vegetable intakes and more likely to consume fast food. To further advance our knowledge about the interplay between time and food, this study examines how time management styles among college students affects their food consumption. Specifically, we examined the impact of four time management styles (short-range planning, long-range planning, time attitude, and preference for organized time) on food choices and eating motives. We found that preference for organized time is positively 
related to healthier food choices and culture (American vs. Chinese) moderates the effect (i.e. the positive effect is stronger among Chinese students than American students). The research also reveals differences in time management styles and eating motives between American vs. Chinese students. Our findings provide theoretical contributions as well as implications for food and beverage manufacturers, consumers, and policy makers.

\section{Hypothesis Development}

Studies in the U.S. have shown that people have increasingly perceived time scarcity (Zuzanek, becker, Peters 1998; Godbey, Lifset, and Robinson 1998). The feeling of not having enough time has been associated with increased employed parents (Daly 1996), working long hours (Godbey et al. 1998), and the fact that most adults have multiple roles such as being parents and employees at the same time (Celnik, Gillspie, and Lean 2012). As people experience greater time scarcity, research in food and health domains revealed an increase in overweight and obesity in adults and children (Cawley 2004; Jeffery and French 1998), an increase in fast food and snack consumption (Gillis and Bar-Or 2003; Jeffery and French 1998), and a decrease of food preparation time (Sayer 2001, Shelton 1992). More than a third of parents in the U.S. reported that they consume takeout food regularly and one-fifth of meals are consumed in a car (Gardyn 2002). The lack of time is reported as a significant barrier to live healthy lifestyle (Furst, Connors, Bisogni, Sobal and Falk 1996).

Based on prior research, this present research proposes that given that time scarcity is a barrier to healthy food choices, individuals who engage in effective time management should be able to have better control over their time and be able to make healthier food decisions than those who cannot manage their time effectively. Our research focuses on college students as they are in an early stage of developing their time management to allow them to handle multiple classes, homework, assignments, parttime jobs, extracurricular activities, and social events. Prior research 
(Britton and Tesser 1991) has studied time management styles among college students by examining short-range vs. long-range planning, and time attitudes (the feeling of spending their time constructively) and found that those who reported greater control of their time also reported greater work and life satisfaction, and greater evaluation of their performance. In the consumer behavior literature, prior research (Usunier and Valette-Florence, 1994) has constructed a similar instrument to measure preference for organized time (e.g. need to establish and follow schedule). Thus, based on the above research findings, this present research hypothesizes that:

H1: Individuals who engage in greater time management (shortrange, long-range planning, time attitude, and preference for organized time) are more likely to choose healthier food choices than those who do not exercise much control over their time.

Furthermore, cross-cultural psychology literature suggests that time perception is a multidimensional construct and it can be influenced by culture. In the U.S., people tend to subscribe to a belief that time is money and because time is a scarce resource, time should be spent wisely. Therefore, norm in the American culture is to be very strict when it comes to time schedules, appointments, and precise setting of duration of an event (Usunier and Valette-Florence, 1994). Prior research (Hall 1983 and Bluedorn 1998) classified this time style that focuses on schedules and finishing one task at a time as monochronic time ( $\mathrm{M}$ time). In contrast, the time style that is more lenient and focuses more on people and events than the time itself is called polychronic time (P time). People who operate in $\mathrm{P}$ time feel more comfortable engaging in multitasking and/or engaging in conversation with multiple people at the same time. $\mathrm{M}$ time is common in American culture while $\mathrm{P}$ time is found more frequently in Middle East, Latin American, and Asian cultures (Usunier and Valette-Florence, 1994).

Since culture may determine the time style adopted by individuals, we believe that culture will moderate the effect of time management 
on food choices. Individuals in Asian cultures tend to use P time and are more willing to adjust their time styles to fit various tasks that they have to accomplish. Since they are quite multitasking, they may be able to simultaneously pursue multiple goals (e.g. healthy goal, career goal, academic goal) in their daily lives without relying on a particular time management style. Thus, we believe that the relationship between time management styles and food choices among Asian college students will be weaker than that among American college students. In other words, we propose that American students are in greater need to have a structured time management in order to maintain their healthy lifestyle.

H2: The positive effect of time management styles (short-range, long-range planning,

time attitude, and preference for organized time) on healthy food choices is more pronounced among American than Asian cultures.

\section{METHOD}

Eighty two American students in the U.S. and thirty-two students in China participated in this study for extra credits. Participants completed an online survey containing questions related to their time management and food consumption. To measure their food choices, the first section of the survey involves a food shopping task in which participants were shown a series of fourteen food and beverage items. For each item, they were asked to decide whether they would purchase it for their own consumption or not (Yes vs. No). Out of the fourteen items, seven were vice and the other seven were virtue foods/beverages. The vice and virtue classification and the food and beverage items were adopted from Thomas, Desai, and Seenivasan (2012). Consumption of vice food and beverages results in immediate gratification (taste good) but does not provide any long-term health benefits whereas consumption of virtue food does not result in immediate gratification but provides long-term health benefits. Vice items are for example donut, cake, hot dog, and 
frozen meals and virtue items are for example salads, yogurt, apple, and whole wheat bread. This food shopping task was given to participants as the first task to prevent any potential biases caused by time management and eating motive measures.

In the next section of the survey, participants evaluated their time attitudes, short-range vs. long-range time management (measures adopted from Britton and Tesser, 1991), and their preference for organized time (measures adopted from Usunier and Valette-Florence, 1994). Next, to deepen our understand of the reasons of their food choices, we measure eating motives by asking participants to complete the Testing of Eating Motivation Survey (TEMS) which was adopted from Renner, Sproesser, Strohbach, and Shupp (2012). The TEMS measures eating motives on multiple dimensions such as liking, habits, hunger, health, price, affect regulation, visual appeal, and weight control. Examples of measures of liking motive are "I eat what I eat because I like it," and "I eat what I eat because I have an appetite for it." After reporting their eating motives, participants was asked "Do you believe 'what you eat' and 'how you manage your time' are related? ( $1=$ not related at all to $7=$ highly related $)$. Lastly they reported their weight, height, GPA, number of sleep hours, exercise behavior, and other demographic information.

\section{Results}

Participants Profile: About 57\% of participants are female. The average age is 20 years old and majority of them are in their senior year in college.

Belief about time management and food consumption: The analysis shows that American participants hold a stronger belief that their time and food consumption are related than Chinese participants $\left(\mathrm{M}_{\text {Americans }}\right.$ $=5.07$ vs. $\mathrm{M}_{\text {Chinese }}=3.72, p<.01$ ). 
26 BRC Journal of Advances in Business Vol. 3, No. 1

\section{Preview Complete}

This completes the limited preview of this paper. Please visit the link below to purchase.

\section{Citation Information}

Gordon, Tal, and Napatsorn Jiraporn. "Time Management Styles and Food Consumption: A Pilot Study of American and Chinese College Students." BRC Fournal of Advances in Business 3, no. 1 (2018): 35-49. http://dx.doi.org/10.15239/j.brcadvjb.2018.03.01.ja03

\section{Web APPEndix}

A web appendix for this paper is available at: http://dx.doi.org/10.15239/ j.brcadvjb.2018.03.01.wa03 